V. REUTRON STAR PHYSICS

CHAIR: T. Weaver 


\title{
NEUTRON STAR INTERIORS
}

\author{
Charles Alcock \\ Department of Physics \\ Massachusetts Institute of Technology, Cambridge, MA 02139 U.S.A.
}

\begin{abstract}
The current status of the theory of neutron star interiors is reviewed. The various phases of matter that might exist are discussed and their relation to other areas of astrophysics briefly noted. Observational distinction between the models is not simply obtained; analysis of pulsar post-glitch relation, of neutron star precession and neutron star cooling are promising in this regard.
\end{abstract}

\section{INTRODUCTION}

This meeting has demonstrated how much our understanding of the properties of neutron stars has grown since their identification in 1968. It is no longer reasonable to describe neutron stars as unusual objects. However, in spite of the great theoretical progress in the past eighteen years, it is still not possible to describe the interior of a neutron star with certainty; indeed, it may turn out that there are no neutrons at all in these objects, and that the title of this meeting is a misnomer! Nevertheless, in spite of the significant conceptual differences between the various models for neutron star interiors, the gross properties of the objects (of mass $1.4 M_{\odot}$ ) are well-described by all the models. Observable differences between the models are subtle.

There have been a number of excellent review articles on neutron star interiors since IAU Symposium No. 53 met in 1972. Prominent among these are the two reviews of Baym and Pethick $(1975,1979)$ and the textbook of Shapiro and Teukolsky (1983). More ambitious recent work on the attempts to use neutron stars as "hadron physics laboratories" was recently reviewed by Pines (1985). This brief review cannot compete in depth with these precedessers; instead, a slightly different slant will be used to illuminate the range of possibilities for descriptions of neutron star interiors.

\section{STATES OF MATTER}

The interior conditions in neutron stars are conventionally described by the physics of "cold, dense matter". This is entirely appropriate, even when finite temperature effects are taken into account (as in the cooling of neutron stars) 
because the total heat content of neutron stars (more than a few seconds after formation) is small compared to their energy density.

It was remarked in the introduction that there is considerable uncertainty regarding the state of the interior of a neutron star. It is informative to explore the physics involved using a phase diagram. Phase diagrams customarily show the material of phase of some substance at all loci in a plane projection of an $(n+1)$-dimensional space where the independent variables are temperature $(T)$, pressure $(P)$, and the $(n-1)$ independent abundances of the $n$ distinct components; the equilibrium phase is obtained by minimizing the Gibbs potential. In principle, our job is easier since only two independent variables, $T$ and the baryon number density $\left(n_{B}\right)$ need be specified; the equilibrium phase may be found by minimizing the free energy. (Minimizing the Gibbs potential at each $(T, P)$ is formally equivalent, but less illuminating in this context).

Of course, most phase diagrams are obtained empirically, usually by materials scientists or geophysicists. Much of the $\left(T, n_{B}\right)$ plane that is of interest to us is inaccessible experimentally, and the diagram is filled out theoretically. There are many sources of uncertainty in this process, which stem from the inherent difficulties in carrying out any strong interaction calculation. The two phase diagrams that are presented here are representative of the uncertainties involved, and in addition, both are consistent with all known facts (i.e. nuclear masses, scattering data).

\section{THE STANDARD MODEL}

In Fig. 1, a phase diagram for the hadrons that will be called the "standard model" is presented. For completeness, the plane includes both "hot" and "cold" regions; specifically, both the temperatures and the chemical potentials range from $\sim 1 \mathrm{KeV}$ to $\sim 1 \mathrm{GeV}$. Except for the possibility discussed in $\S 4$, all of the features in Fig. 1 are very likely to be correct. The precise locations of the boundaries are not at all certain, especially in the hot, dense region; however, the existence of these boundaries and the phases that are illustrated is reasonably well-established.

At temperatures below $3 \times 10^{9} \mathrm{~K}$ and densities below $8 \times 10^{6} \mathrm{~g} \mathrm{~cm}^{3}$, the equilibrium phase is ionized ${ }^{56} \mathrm{Fe}$. At higher temperatures thermodynamic equilibrium shifts in favor of lighter nuclei (shaded region), and for temperatures in the range $10^{10} \mathrm{~K}$ to $10^{12} \mathrm{~K}$ we find protons, neutrons, and electrons, with most of the energy in photons and neutrinos. These composition changes are gradual; no phase transitions occur in this region.

Somewhat near $T=2 \times 10^{12} \mathrm{~K}$ a dramatic phase transition between nucleonic matter and free quarks occurs. The existence of this phase transition is more or less assured; this statement depends on the existence of quarks as the fundamental constituents of nucleons and the property of asymptotic freedom (i.e. that at high energies the quark-quark interactions are not strong.) There is some evidence that this is a first order phase transition. There is good reason to believe it occurs near $T \sim 2 \times 10^{12} \mathrm{~K}$, but a precise calculation of the transition temperature is not 


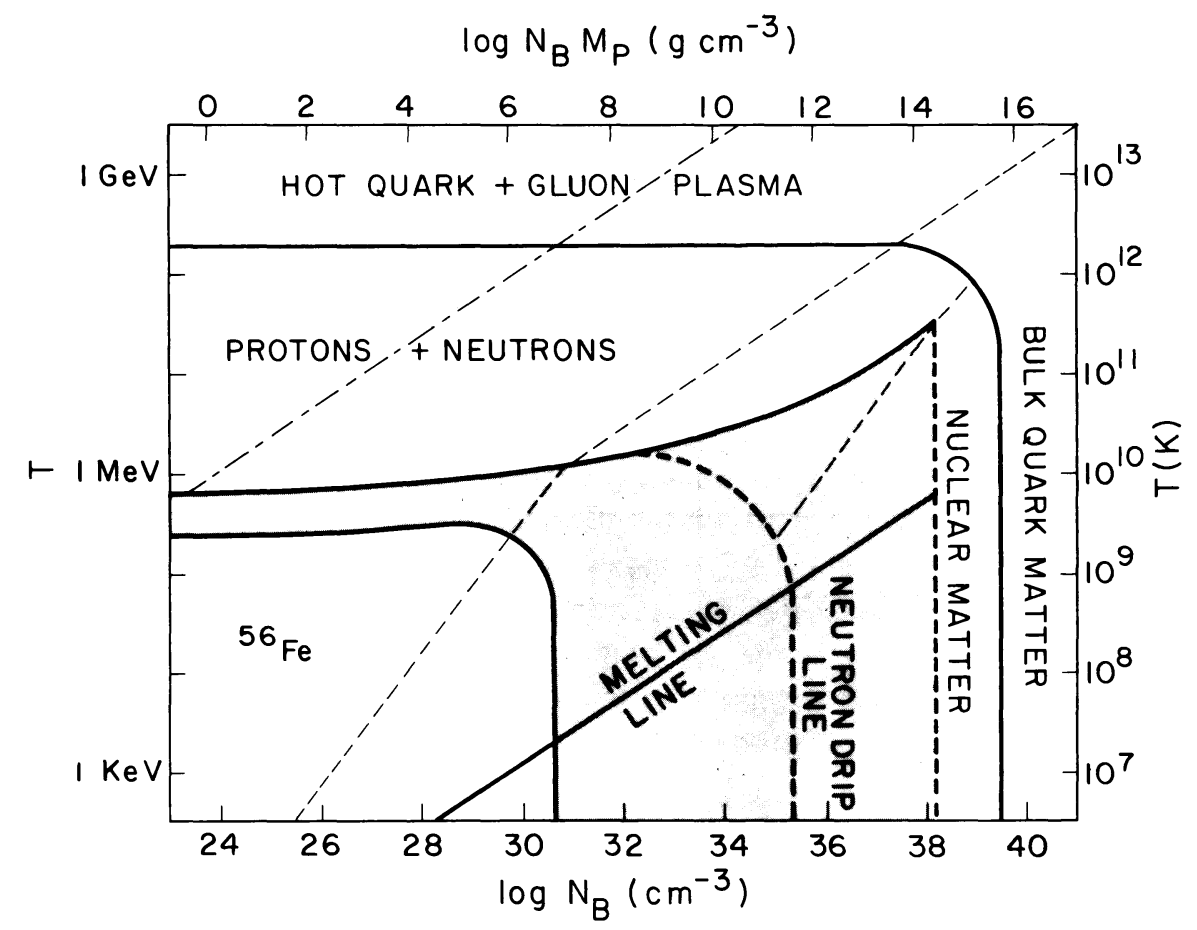

Fig. 1: Phase diagram for the hadrons in the standard model. The regions in which the hadrons exist as ${ }^{56} \mathrm{Fe}$, as protons and neutrons, or as free quarks, are marked. In the shaded area, intermediate nuclei are found: at $n_{B} \lesssim 10^{30} \mathrm{~cm}^{-3}$ these are nuclei lighter than ${ }^{56} \mathrm{Fe}$, while in the higher density shaded region heavier nuclei are found. First order phase transitions separate the quark phase from the nucleonic phase and the solid ionic lattice from the liquid ionic phase (melting line). The neutron drip line and nuclear density line are marked. The long dashed line marks the onset of electron degeneracy. The shorter dashed line marks the onset of neutron degeneracy. The universe evolved along the dot-dashed line.

possible at present. This is not the place to review these issues carefully; access to the literature may be made through Hogan (1983) and Witten (1984).

The universe evolved through the hot, low density portion of Fig. 1; however, for kinetic reasons the universe went out of thermodynamic equilibrium as it cooled below $10^{10} \mathrm{~K}$, which is why the universe today is not made of lumps of iron.

Now consider the phase plane for $T \gtrsim 3 \times 10^{9} \mathrm{~K}$ and for densities increasing up to $4 \times 10^{11} \mathrm{~g} \mathrm{~cm}^{-3}$. When the electron degeneracy line is crossed, the material may be described as "cold". Several interesting things occur in this region, which represents the "outer crust" of a neutron star. This discussion is based on that of Baym, Pethick and Sutherland (1971). First, when the melting line is crossed, the 
ions arrange themselves into a solid lattice; this is a first order phase transition, but very little latent heat is evolved. Second, the increasing electron potential induces electron captures, altering the equilibrium nuclide from ${ }^{56} \mathrm{Fe}$ at low densities through eleven steps up to ${ }^{118} \mathrm{Kr}$. Each change in equilibrium nuclide is a first order phase transition. It is interesting to note that the equilibrium nuclide at a given pressure is determined, in part, by the lattice contribution to the Gibbs potential.

Continuing at low temperatures but to higher densities, one encounters at $4.1 \times 10^{11} \mathrm{~g} \mathrm{~cm}^{-3}$ the phenomenon of "neutron drip". Free neutrons appear in the system. As the density increases the abundance of neutrons increases, and the equilibrium nuclide shifts toward superheavy, very neutron rich species which are arranged in a solid lattice. The equation of state for the region, which represents the "inner crust" of a neutron star, has been discussed by Baym, Bethe and Pethick (1971), Negele and Vautherin (1972) and Negele (1974), among others. Recent work on the superfluid behavior of the neutrons in this region has been reviewed by Pines and Alpar (1985) and by Pines (1985).

At nuclear density $\left(2.8 \times 10^{14} \mathrm{~g} \mathrm{~cm}^{-3}\right)$ and above, the identity of individual nuclei is clearly lost. This material is an interacting gas comprising neutrons with a smaller number of protons and electrons; at densities above a few times nuclear $\Sigma^{-}$and then $\Lambda$ hyperons appear, but do not significantly affect the equation of state.

While there is general agreement on the constituent particles in this density regime (but see the discussion in $\S 4$ for some disagreement!), there is a wide variety of proposed equations of state for this region. This variety reflects the inherent difficulties of nuclear many-body theory, in that a good model for the interaction potential does not exist, and further that the computational techniques needed to construct an equation of state are not fully developed. There is insufficient space here to appraise the literature critically, and instead representative results will be presented for (a) the mean field (MF) theory calculation of Pandharipande and Smith (1975a); (b) the tensor interaction (TI) model of Pandharipande and Smith (1975b); and (c) the pure neutron gas with Reid potential (R) (Pandharipande 1971). MF and TI are "stiff" models and $R$ is a "soft" model.

At the high density end of the nuclear matter region the electron chemical potential may rise above the effective mass for the negative pion, resulting in the appearance of pions and the possibility of a Bose condensation of pions. This condensation adds massive particles into states of low momentum, thus softening the equation of state. Additionally, the pions greatly enhance the cooling rate due to neutrino emission. Pandharipande (1971) has computed a model equation of state $(\mathrm{P})$ using a Reid potential and a low enough pion effective mass that the condensation appears at a density of $6 \times 10^{14} \mathrm{~g} \mathrm{~cm}^{-3}$.

Since it is clear that neutrons and protons are comprised of quarks, and further that the nucleons have finite sizes, it is obvious that at some density the identity of individual nucleons is lost and that a bulk quark phase results. The 
existence of a phase transition of this kind is not in doubt, however, the density at which this transition occurs is not known. If the transition occurs at a density of $\sim 10^{15} \mathrm{~g} \mathrm{~cm}^{-3}$, neutron stars would have a core of quark matter: such stars are known as "quark stars" and have been discussed by, among others, Chapline and Nauenberg (1977), and Fechner and Joss (1978). The uncertainties involved in a discussion of quark matter are discussed in $\S 4$.

It is worth remarking on the different natures of the uncertainties regarding the existence of quark matter and of pion condensates. There is no doubt that above some density, quark matter exists; there is considerable doubt about whether it exists in neutron stars. On the other hand, while a pion condensate will certainly form in a gas of nucleons above some density, it is possible that quark matter forms out of nucleons before that density is reached; there is, therefore, real doubt about whether pion condensates exist under any conditions.

The high temperature, high density region of Fig. 1 is the least certain portion of the phase plane; the various boundaries were joined together in a plausible fashion. This region of the plane appears to have little interest to astrophysics, except perhaps to model neutron star collisions. It is this region that will be explored in relativistic heavy ion collisions.

\section{STRANGE MATTER}

A radically new possibility regarding the equilibrium state of matter has been raised by Witten (1984). Witten proposed that the true ground state of the hadrons is "strange matter", not ${ }^{56} \mathrm{Fe}$. Strange matter is a bulk quark phase that consists of roughly equal numbers of up, down and strange quarks, plus a smaller number of electrons (to guarantee charge neutrality) which is conjectured to have a lower energy per baryon than ordinary nuclei. This means that strange matter is absolutely stable at zero pressure.

A phase diagram that is consistent with this picture is shown in Fig. 2. At densities $\gtrsim 4 \times 10^{14} \mathrm{~g} \mathrm{~cm}^{-3}$ and temperatures $\lesssim 10^{11} \mathrm{~K}$, there is bulk strange matter, which in many respects is similar to the quark matter phase discussed above. What is different here is that the pressure of strange matter vanishes at $\rho \approx 4 \times 10^{14} \mathrm{~g} \mathrm{~cm}^{-3}$. If the mean baryon density of the universe falls below this value, the equilibrium consists of a gas of lumps of strange matter (strangelets), each of which have internal densities of $4 \times 10^{14} \mathrm{~g} \mathrm{~cm}^{-3}$. These lumps may have baryon numbers between $\sim 100$ and $2.5 \times 10^{57}$; the upper limit is set by gravitational collapse. The pressure of this phase is entirely due to the photons and neutrinos.

There is a region in the phase plane where neutrons and protons are the thermodynamic equilibrium. This occurs for the same reason that ${ }^{56} \mathrm{Fe}$ is not favored in this region: strange matter is a minimum of the energy, but not of the free energy.

As in the standard model discussed in $\S 3$, a first order phase transition between nucleons and free quarks occurs at $T \sim 2 \times 10^{12} \mathrm{~K}$. Note that the universe 


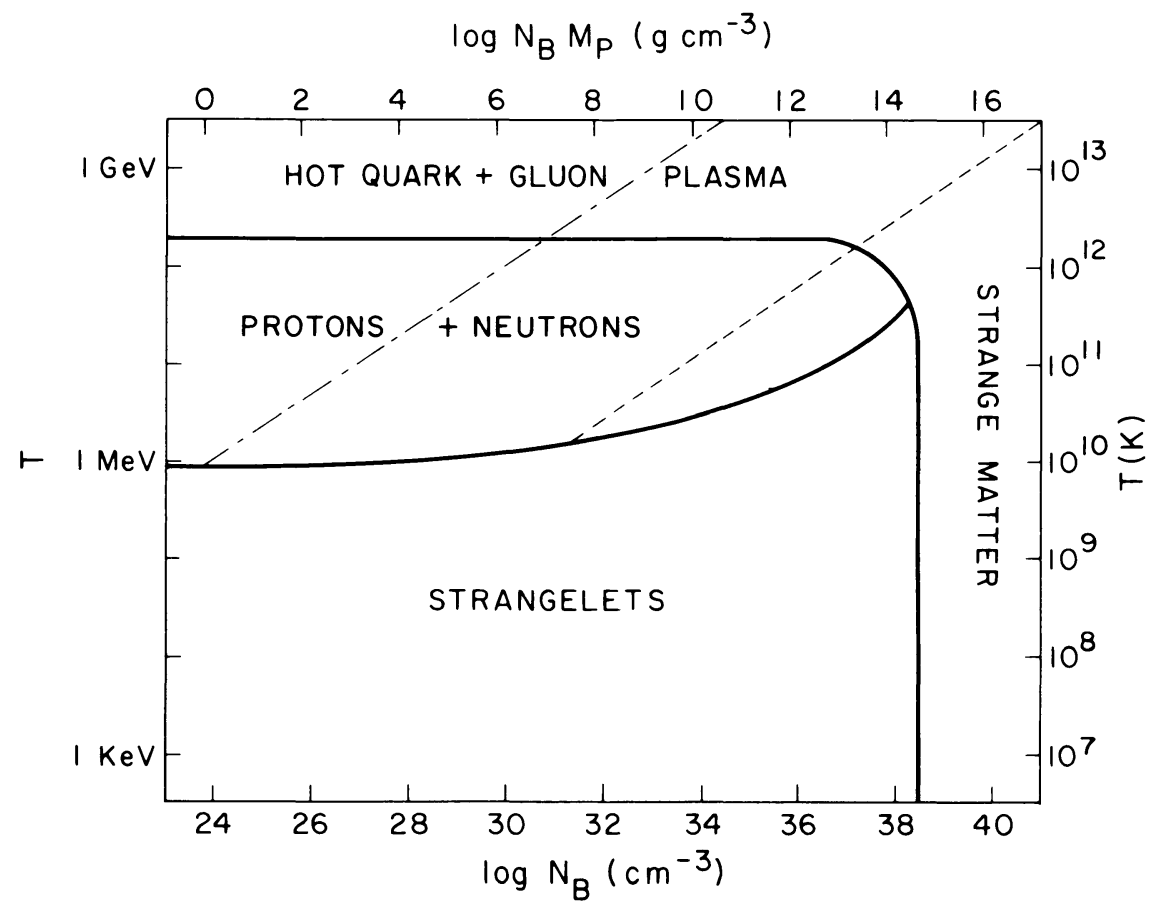

Fig. 2: Non-standard phase diagram for the hadrons. The hadrons exist either as a gas of strangelets, as neutrons and protons, or as free quarks. The neutrons and protons are separated from the quark phase by a first order phase transition. The dashed line marks the onset of electron degeneracy; note that there are almost no free electrons in the gas of strangelets. The universe evolved along the dot-dashed line.

evolves through the "neutron and proton" region; it has been shown that any strangelets which form during the phase transition (a possibility proposed by Witten (1984) and disputed by Applegate and Hogan (1985)) evaporate completely into protons and neutrons, which means that there is very little cosmic strange matter today (Alcock and Farhi 1985).

It is puzzling, given the long history of discussions of quark matter, that the strange matter hypothesis was made only recently. Various forms of quark matter have been discussed by, among others, Ivanenko and Kurdgelaidze (1969), Itoh (1970), Collins and Perry (1975), Freedman and McLerran (1978), Baluni (1978), and Chin and Kerman (1978). The possibility that a form of quark matter with a significant fraction of strange quarks might be absolutely stable was raised by Witten (1984). A detailed study by Farhi and Jaffe (1984) showed that, with the uncertainties inherent in a strong interaction calculation, the existence of strange matter is reasonable. This means that no decision can be made at this point about which of Figs. 1 and 2 represents the equilibrium states of the hadrons. It then behooves us to examine the consequence of this phase for neutron stars; this 
has been done by Haensal, Zdunik and Schaeffer (1985), Baym et al. (1985) and by Alcock, Farhi and Olinto (1986).

Since strange matter is absolutely stable, a star may be made entirely of strange matter. Such an object (a "strange star") has an exposed quark matter surfaces which, since it is held together by the strong force, is not subject to the Eddington limit. However, this surface is probably highly reflective in the Xray (Alcock, Farhi and Olinto (1986), and very high luminosities will only occur in very hot, transient events. Furthermore, the star does not necessarily have an exposed quark matter surface, since a layer of material identical to the outer crust of a neutron star can be supported electrostatically above the quark surface. There is no possibility of an inner crust in a strange star because the strange matter absorbs all the free neutrons; this may have important consequences for the interpretation of observations, as will be discussed in $\S 6$.

\section{GLOBAL PROPERTIES OF NEUTRON STARS}

It is well-known how to construct models of compact stars (see e.g. Shapiro and Teukolsky 1983). The vast amount of work in this area is summarized in Fig. 3 which shows mass-radius relations for models constructed with the various equations of state for nuclear matter that were discussed in $\S 3$, and for the strange matter equation of state (S) that was derived by Alcock, Farhi and Olinto (1986).

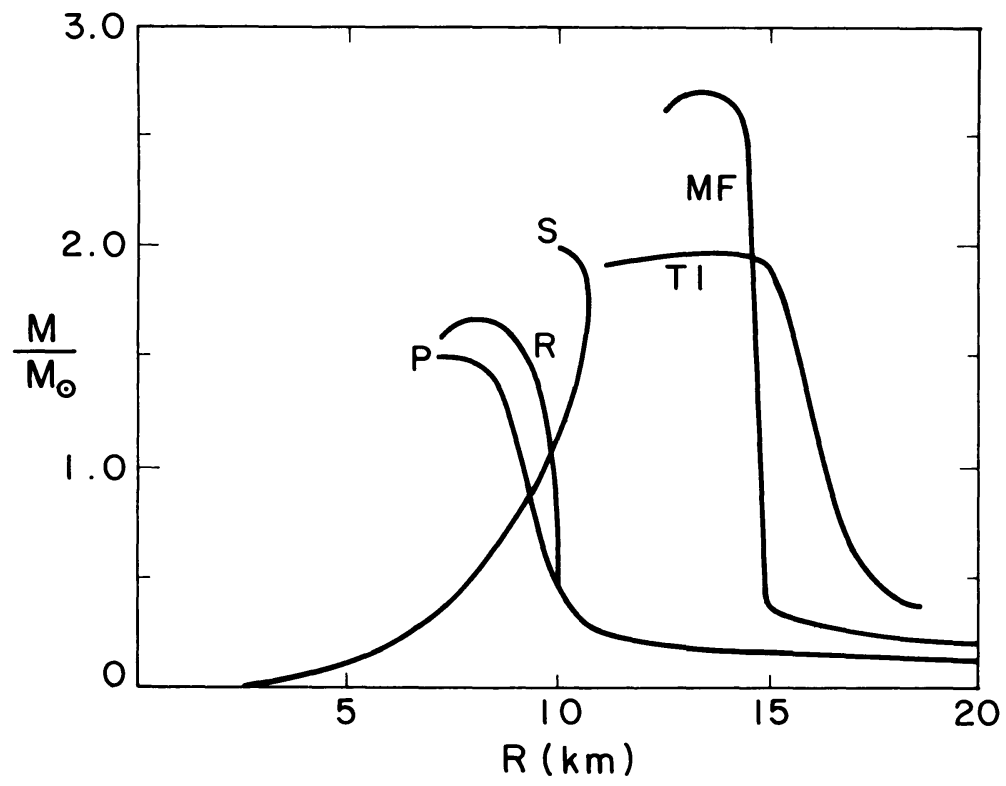

Fig. 3: Mass vs. radius for four neutron star equations of state and for one strange star equation of state. 
Most of this figure (i.e. the curves for the four nuclear matter equations of state) is familiar. The strange star mass-radius relation is very different. For low masses, the stars have uniform density and $M \propto R^{3}$; the minimum mass is $\sim 10^{-55} M_{\odot}$. As the mass increases, gravity becomes important and there is a maximum radius of $\sim 11 \mathrm{~km}$, followed by a maximum mass $\sim 2 M_{\odot}$.

However different the strange star mass radius relation is overall, in the mass range that matters $\left(\sim 1.4 M_{\odot}\right)$ it is not greatly different from a neutron star with a Reid potential equation of state. In fact, any of the mass-radius relations shown in Fig. 3 can be reconciled with the observational data on neutron stars; this reflects our ignorance of neutron star radii.

\section{DISTINGUISHING THE MODELS}

A decision between the models for neutron star interiors may be made on the basis of improved theory. Alternatively, a decision may be reached by analysis of observations of neutron stars, an approach advocated by Pines (1985). The observational distinctions may arise from analysis of pulsar glitches, from evidence of neutron star precession, or from analysis of thermal X-rays from cooling neutron stars.

There is a theory for pulsar post-glitch relaxation that involves the massive uncoupling of neutron superfluid vortices in the inner crust during a glitch, followed by a gradual re-coupling of the vortices to the charged component in the star, and thus to the magnetic field. This theory, which has been reviewed by Pines and Alpar (1985) and by Pines (1985), is attractive in that a natural explanation of the long post-glitch relaxation time is possible.

Since all the action in the neutron superfluid model for post-glitch relaxation occurs in the inner crust, it is useful to see how this portion of the star depends on the equation of state in the interior. The radial fractions of core, inner crust and outer crust for three models of $1.4 M_{\odot}$ stars are shown in Fig. 4. Note that the stiff (TI) model has a substantial inner crust, the soft (R) model much less, and the strange star (S) has none. Clearly, the neutron superfluid model does not apply to the strange star picture; if no model for glitches is developed in that picture, the success of the neutron superfluid models could be interpreted as evidence against the strange matter hypothesis. In this fashion, which unfortunately is model dependent, observations of neutron stars may shed light on fundamental physics.

Another model dependent statement regarding the inner crust has been made recently. Trumper et al. (1986) interpret that EXOSAT data on the 35-day cycle in Hercules X-1 as evidence for large amplitude $\left(\sim 25^{\circ}\right)$ free precession of the neutron star. This model requires a rather thick inner crust, which leads Pines (1985) to argue that the equations of state must be comparatively stiff.

The final remark in this section concerns the cooling of neutron stars. There is evidence for a contradiction between X-ray observations of young supernova rem- 


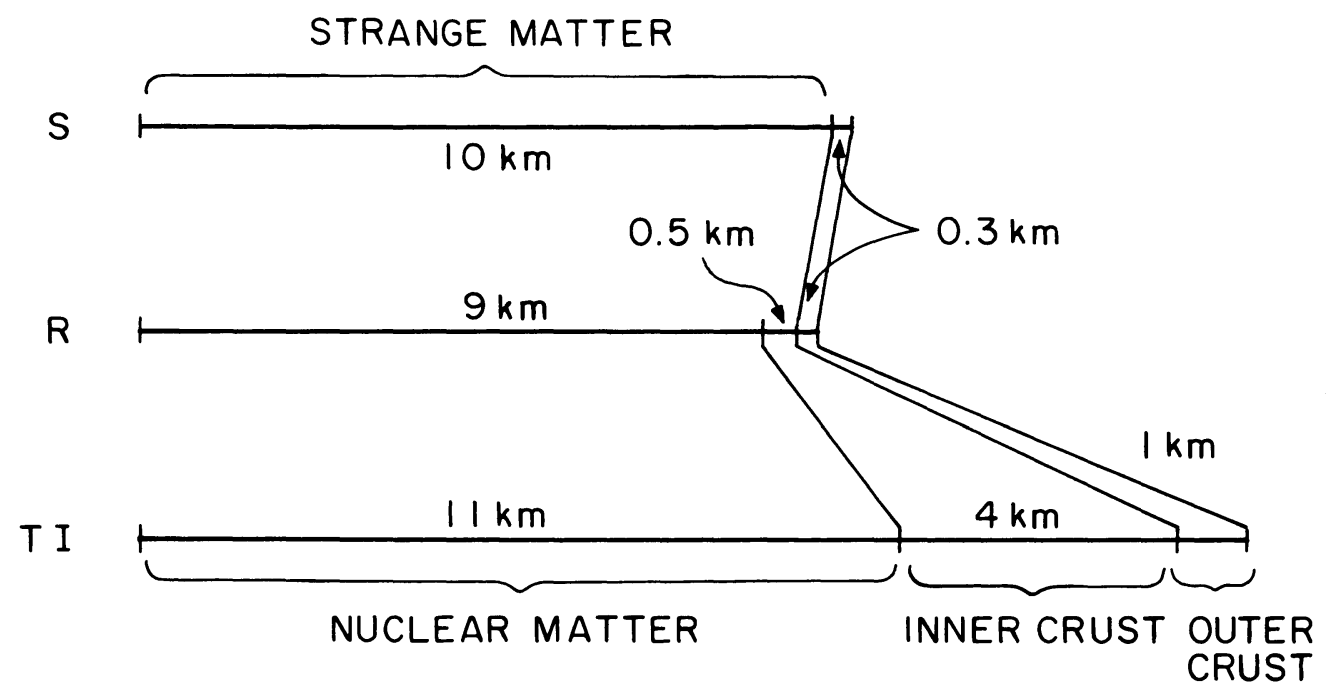

Fig. 4: Schematic showing the relative proportions of the core, the inner crust, and the outer crust for three models of a $1.4 M_{\odot}$ object.

nants and the cooling of neutron stars in the standard models (see e.g. Glen and Sutherland 1980; Van Riper and Lamb 1981; Yakovlev and Urpin 1981; Nomoto and Tsuruta 1981). Stars with pion condensates cool much faster (Maxwell et al. 1977) and so do stars containing quark matter (see e.g. Iwamoto 1980; Duncan, Wasserman and Shapiro 1983; Alcock, Farhi and Olinto 1986). Should unambigious evidence for "cool, young" neutron stars come available, this could naturally be interpreted as evidence for pion condensates or quark matter.

In summary, it is possible in principle to draw observational distinctions between the various models of neutron stars. However, such distinctions are often highly model dependent, and we may be testing the imagination of astrophysicists as much as the hadron physics.

\section{CONCLUSIONS}

A "safe" conclusion is that neutron star interiors remains a fascinating area of research. It is not possible to conclude firmly what are the microscopic constituents of a neutron star; there may even be no neutrons in a neutron star.

The best prospects for deciding between the competing models appears to be in the analysis of pulsar glitches, and the study of the dynamics of precessing neutron stars may also prove fruitful; in both of these cases the conclusions will inevitably be model dependent. X-ray observations of young neutron stars are potentially a less model dependent tool. 


\section{REFERENCES}

Alcock, C., and Farhi, E. 1985, Phys. Rev. D, 32, 1273.

Alcock, C., Farhi, E., and Olinto A. 1986, to appear in Ap. J.

Applegate, J., and Hogan, C. 1985 Phys. Rev. D., 31, 3037.

Baluni, V. 1978, Phys. Lett., 72B, 381.

Baym, G., Bethe, H. A., and Pethick, C. J. 1971, Nucl. Phys., A175, 225.

Baym, G., Jaffe, R. L., Kolb, E. W., McLerran, L., and Walker, T. P. 1985, Phys. Lett., 160B, 181.

Baym, G., and Pethick, C. J. 1975, Ann. Rev. Nucl. Sci., 25, 27.

Baym, G., and Pethick, C. J. 1979, Ann. Rev. Astr. Ap., 17, 415.

Baym, G., Pethick, C. J., and Sutherland, P. G. 1971, Ap. J., 170, 299.

Chapline, G., and Nauenberg, N. 1977, Phys. Rev. D, 16, 450.

Chin, S., and Kerman, A. 1978, Phys. Rev. Lett., 43, 1292.

Collins, J., and Perry, M. J. 1975, Phys. Rev. Lett., 34, 1353.

Duncan, R. C., Shapiro, S. L., and Wasserman, I. 1983, Ap. J., 267, 358.

Farhi, E., and Jaffe, R. L. 1985, Phys. Rev. D., 32, 2452.

Freedman, B., and McLerran, L. 1978, Phys. Rev. D, 17, 1109.

Glen, G., and Sutherland, P. 1980, Ap. J., 239, 671.

Haensel, P., Zdunik, J. L., and Schaeffer, R. 1985, preprint.

Hogan, C. J. 1983, Phys. Rev. Lett., 51, 1488.

Itoh, N. 1970, Prog. Theor. Phys., 44, 291.

Ivanenko, D., and Kurdgelaidze, D. F. 1969, Lett. Nuovo Cimento, 2, 13.

Iwamoto, N. 1980, Phys. Rev. Lett., 44, 1637.

Maxwell, O., Brown, G. E., Campbell, D. K., Dashen, R. F., and Manassah, J. T. 1977, Ap. J., 216, 77.

Negele, J. W. 1974, in Physics of Dense Matter, ed., C. J. Hansen (Dordrecht:Reidel), 1.

Negele, J. W., and Vautherin, D. 1972, Phys. Rev. C., 5, 1472.

Nomoto, K., and Tsuruta, S. 1981, Ap. J. (Letters), 250, L19.

Pandharipande, V. R. 1971, Nucl. Phys. A, 178, 123.

Pandharipande, V. R., and Smith, R. A. 1975a, Nucl. Phys. A., 237, 507.

Pandharipande, V. R., and Smith, R. A. 1975b, Phys. Lett., 59B, 15.

Pines, D. 1985, Lectures presented at NATO Institute "High Energy Phenomena and Collapsed Stars".

Pines, D., and Alpar, M. A. 1985, Nature, 316, 27.

Shapiro, S. L., and Teukolsky, S. A. 1983, "Black Holes, White Dwarfs, and Neutron Stars" (Wiley: New York).

Trümper, J., Kahabka, P. Ögelman, H., Pietsch, W., and Voges, W. 1986, Ap. J. (Letters), 300, L63.

Van Riper, K. A., and Lamb, D. Q. , Ap. J. (Letters), 244, L13.

Witten, E. 1984, Phys. Rev. D, 30, 272.

Yakovlev, D. G., and Urpin, V. A., Soviet Astr. Letters, 7, 88. 


\section{DISCUSSION}

F. Frontera: Can the period noise observed in X-ray pulsars be interpreted as due to the interval structure of "Strange Stars"?

C. Alcock: Probably not, since the strange matter behaves like a Newtonian fluid; however, we have not looked at this issue carefully. Of course, the period noise may be related to the accretion process.

S. Colgate: Wang and Eichler point out that a dipole magnetic field's decay is dependent upon the crustal conductivity. The interior fluid allows multipoles to form. Would not a strange star with no crust allow the rapid decay of the presumed dipole field?

C. Alcock: My guess is that the answer is yes, but I have not yet fully understood the calculation of Wang and Eicher. I should point out that the maximum crust allowed on a strange star is modest compared to that of a neutron star, so that in any event the Wang and Eichler mechanism should proceed rapidly.

J. Shaham: Is there enough crust in "strange stars" to still accomodate Bisnovatyi-Kogan's $\gamma$-ray burst mode1?

C. Alcock: I don't think so, since much of the physics in that model occurs deep inside the inner crust.

M. Bailes: Is strangeness conserved and what do you make a strange quark out of?

C. Alcock: Strangeness is not conserved in the weak interaction. Strange quarks may be made in a number of reactions, the most important being the reaction $u+d \rightarrow u+s$.

A. Burrows: Please describe the processes involved in the propagation of the strange front to the surface and give an estimate of its velocity.

C. Alcock: A strangeness front absorbs neutrons, liberating $u$ and $d$ quarks behind the front. The equilibration with $s$ quarks is achieved by both weak interactions and diffusion of $s$ quarks from behind the front. As a result, the front moves about one strong interaction length per weak interaction time. We have not calculated this yet, but the velocity may be as slow as $1 \mathrm{~mm} / \mathrm{s}$.

v. Trimble: Can you predict the decay time for a magnetic field anchored in a quark star rather than a neutron star?

c. Alcock: We have not done a decent calculation yet. The result may be very similar to that for a neutron star. The quark fluid may become a superconductor, a possibility that is still being investigated.

J. Taylor: Is there a simple-minded explanation for what seems to me to be an astonishing fact: that your strange stars have the same masses and radii as the neutron stars that we all know and love?

C. Alcock: This is because the natural density scale for strange matter $\left(4 \times 10^{14} \mathrm{~g} \mathrm{~cm}^{-3}\right)$ is close to the mean density for a neutron star. The neutron star achieves this mean density only very close to its maximum mass, which is why the mass-radius relations are so similar here. 
N. Itoh: In your talk you mentioned the increase of the electron chemical potential as the main cause of the appearance of the pion condensate. I believe the pion-nucleon interaction is essential in bringing about the pion condensate.

C. Alcock: Maybe I was unclear on this point. The true condition for the appearance of pions is that the electron chemical potential be larger than the lowest energy state for the pions. The pion-nucleon interaction is crucial in lowering the energy of this state. I had intended to convey this by referring to the "effective mass" for the pion.

G. Bisnovatyi-Kogan: The pion-condensation is connected with the strong interaction between nucleons and not related to the fermi energy of electrons. At densities close to nuclear the potential hole is so deep, that $\pi^{\circ}$ and $\pi^{+} \pi^{-}$pair birth is energetically favored. This process is similar to the birth of $e^{+} e^{-}$pairs in a strong electrical field.

C. Alcock: This is the same as Itoh's question, which I have answered. The pion chemical potential is equal to the electron chemical potential, which is crucial in this issue. 